\title{
Bile acids are physiological ligands for a nuclear receptor
}

Makishima M, Okamoto AY, Repa JJ, et al. Identification of a nuclear receptor for bile acids. Science 1999;284:1362-5.*

\begin{abstract}
Bile acids are essential for the solubilization and transport of dietary lipids and are the major products of cholesterol catabolism. Results presented here show that bile acids are physiological ligands for the farnesoid $X$ receptor (FXR), an orphan nuclear receptor. When bound to bile acids, FXR repressed transcription of the gene encoding cholesterol 7 alpha-hydroxylase, which is the rate-limiting enzyme in bile acid synthesis, and activated the gene encoding intestinal bile acid-binding protein, which is a candidate bile acid transporter. These results demonstrate a mechanism by which bile acids transcriptionally regulate their biosynthesis and enterohepatic transport.
\end{abstract}

\section{Comment}

In May 1999 three papers were published which should change the way we think about bile acids. ${ }^{1-3}$ For long appreciated as detergents, important for micelle formation and lipid absorption in the intestine, they should now also be regarded as members of the steroid family of hormones, affecting transcriptional control of gene expression through a specific nuclear receptor, the farnesoid X receptor.

The primary bile acids (cholate and chenodeoxycholate) are synthesised from cholesterol in the liver with the enzyme cholesterol 7 $\alpha$-hydroxylase (CYP7A) being the rate-limiting step in the classic pathway. The activity of this enzyme has been known for some time to be under inhibitory feed-back control by bile acids. ${ }^{4}$ In view of the importance of this pathway in the removal of cholesterol, the mechanisms for the positive and negative regulation of transcription of the CYP7A gene have been studied. ${ }^{5}$ Until now, the mediator of the effects of bile acids had not been found.

In the ileum, conjugated bile salts are reabsorbed, undergoing an enterohepatic circulation. Again this pathway is regulated by the bile salts themselves. At the cellular level, bile acid absorption involves at least two specific carrier proteins. The ileal apical membrane sodium dependent bile acid transporter (ASBT, also called IBAT), gene name SLC10A2, makes use of the $\mathrm{Na}^{+}$gradient to cotransport conjugated bile acids into the enterocyte. ${ }^{6}$ The second carrier is the smaller $(14-15 \mathrm{kDa})$ soluble protein which binds the absorbed bile acid in the cytoplasm and is now usually termed ileal bile acid binding protein

*See also Parks DJ, Blanchard SG, Bledsoe RK, et al. Bile acids: natural ligands for an orphan receptor. Science 1999;284:1365-8; and Wang H, Chen J, Hollister K, et al. Endogenous bile acids are ligands for the nuclear receptor FXR/BAR. Mol Cell 1999;3:543-53.
(IBABP). ${ }^{7}$ Promoter sequences in mouse, ${ }^{8}$ rabbit and recently the human gene ${ }^{9}$ (Barley NF et al, manuscript submitted) have been characterised.

Regulated expression of these ileal bile acid transporters occurs in rats and in human cell line model systems. IBABP expression was induced in rats by various bile salts in an ileal bypass model, in explants of ileum and in human Caco-2 cells. ${ }^{11}$ Similarly, the apical membrane transporter, ASBT, was increased in rats by feeding cholic acid and reduced by bile diversion. ${ }^{12}$

The mechanism underlying many of these actions is now apparent. Using constructs of the mouse IBABP gene transfected into monkey kidney CV1 cells, Makishima et al were able to identify a DNA sequence in the promoter which functioned as a bile acid response element (BARE). ${ }^{1}$ This was specifically activated by the farnesoid $\mathrm{X}$ receptor and was dependent on the presence of bile acids to act as ligands. The response was greatest with chenodeoxycholic acid (CDCA) - cholic acid was much less effective than CDCA and the secondary bile acids deoxycholic and lithocholic acids had variable activity. Effects were seen with conjugated bile salts in vivo only when the membrane transporter ASBT was also expressed, allowing uptake into the cell. ${ }^{2}$ The interaction in vitro of the farnesoid $\mathrm{X}$ receptor with a steroid receptor coactivator was increased by bile salts-again CDCA at micromolar concentrations was the most specific. ${ }^{23}$

The farnesoid $\mathrm{X}$ receptor binding site in the mouse IBABP promoter, namely the BARE, is an inverted hexanucleotide repeat, spaced by one nucleotide (IR-1). ${ }^{1}$ This sequence is conserved in the rabbit and human genes (Barley NF et al, manuscript submitted). The IR-1 BARE also binds the retinoid $\mathrm{X}$ receptor, so is similar to other members of the steroid hormone receptor family, which includes thyroid hormone, retinoic acid, and vitamin D receptors. Ligand specificity is determined by the spacing of the repeated elements. ${ }^{13}$ There is a large number of nuclear receptors with similar structure in this family (perhaps as many as 200) and until their ligands are known they are called orphan receptors. ${ }^{14}$ Hitherto the farnesoid X receptor had been thought to bind certain cholesterol precursors including farnesol itself. ${ }^{15}$ Other members of this family include hepatocyte nuclear factor 4 (HNF4), apolipoprotein regulatory protein 1 (ARP1), and chicken ovalbumin upstream promoter transcription factor 1 (COUPTF1). Functional ligands are emerging for perioxisomal proliferator activated receptor (PPAR), which binds fatty acids, liver X receptor (LXR $\alpha$ ), which binds oxysterols, and the pregnane $\mathrm{X}$ receptor (PXR), which binds several agents including rifampicin.

In this study, the promoter of the cholesterol synthesis enzyme, CYP7A, was also repressed by CDCA and, at higher concentrations, by other bile acids in the presence of the farnesoid $\mathrm{X}$ receptor but not other nuclear orphan receptors. ${ }^{1}$ Thus bile acids function through this receptor at two key areas, to inhibit their synthesis from cholesterol and, in all likelihood, to promote their re-uptake in the ileum mediated by IBABP. This has wide potential for the targeting of pharmaceutical agents to alter cholesterol lev- 
els and will also have wide relevance for gastroenterology. As always one answer provokes more questions. Will it be possible to induce bile salt re-uptake selectively? Are any of the pathological effects of bile salts mediated by the farnesoid $\mathrm{X}$ receptor? Is the colorectal cancer promoting effect of bile mediated by the receptor? Does this have any relevance for gallstone disease? The farnesoid $\mathrm{X}$ receptor seems likely to be the subject of much further study.

Gastroenterology Section,

J R F WALTERS

Division of Medicine,

Imperial College School of Medicine,

Hammersmith Hospital, London W12 ONN, UK

email: julian.walters@ic.ac.uk

1 Makishima M, Okamoto AY, Repa JJ, et al. Identification of a nuclear receptor for bile acids. Science 1999;284:1362-5.

2 Parks DJ, Blanchard SG, Bledsoe RK, et al. Bile acids: natural ligands for an orphan receptor. Science 1999;284:1365-8

3 Wang H, Chen J, Hollister K, et al. Endogenous bile acids are ligands for the nuclear receptor FXR/BAR. Mol Cell 1999;3:543-53.

4 Russell DW, Setchell KDR. Bile acid biosynthesis. Biochemistry 1992; 31:4737-49.
5 Crestani M, Sadeghpour A, Stroup D, et al. Transcriptional activation of thecholesterol 7alpha-hydroxylase gene (CYP7A) by nuclear hormone thecholesterol 7alpha-hydroxylase gene
receptors. F Lipid Res 1998;39:2192-200.

6 Oelkers P, Kirby LC, Heubi JE, et al. Primary bile acid malabsorption caused by mutations in the ileal sodium-dependent bile acid transporter gene (SLC10A2). 7 Clin Invest 1997;99:1880-7.

7 Oelkers P, Dawson PA. Cloning and chromosomal localization of the human ileal lipid-binding protein. Biochim Biophys Acta 1995;1257:199-202.

8 Crossman MW, Hauft SM, Gordon JI. The mouse ileal lipid-binding protein gene: a model for studying axial patterning during gut morphogenesis. $\mathcal{F}$ Cell Biol 1994;126:1547-64

9 Chakravarty P, Howard A, Shaw-Smith CJ, et al. The human ileal bile acid binding protein gene promoter. Gut 1997;41(suppl 1):A33.

10 Kanda T, Niot I, Foucaud L, et al. Effect of bile on the intestinal bile-acid binding protein (I-BABP) expression. In vitro and in vivo studies. FEBS Lett 1996;384:131-4.

11 Kanda T, Foucand L, Nakamura Y, et al. Regulation of expression of human intestinal bile acid-binding protein in Caco-2 cells. Biochem $\mathcal{F}$ 1998;330: 261-5.

12 Stravitz RT, Sanyal AJ, Pandak WM, et al. Induction of sodium-dependent bile acid transporter messenger RNA, protein, and activity in rat ileum by cholic acid. Gastroenterology 1997;113:1599-608

13 Umesono K, Murakami KK, Thompson CC, et al. Direct repeats as selective response elements for the thyroid hormone, retinoic acid, and vitamin $\mathrm{D}_{3}$ receptors. Cell 1991;65:1255-66.

14 Gustafsson JA. Seeking ligands for lonely orphan receptors. Science 1999;284:1285-6.

15 Forman BM, Goode E, Chen J, et al. Identification of a nuclear receptor that is activated by farnesol metabolites. Cell 1995;81:687-93. 